\title{
Double Lipschitz Stability for Nonlinearly Perturbed Differential Systems with Multiple Delay
}

\author{
Ran Huo ${ }^{1}$, Xiaoli Wang ${ }^{*}$ \\ ${ }^{1}$ College of Science, Inner Mongolia Agricultural University, Huhhot, China \\ ${ }^{2}$ College of Statistics and Mathematics, Inner Mongolia University of Finance and Economics, Huhhot, China \\ Email: huoran124@163.com, ${ }^{*}$ wangxiaoli191898@sina.com
}

How to cite this paper: Huo, R. and Wang, X.L. (2019) Double Lipschitz Stability for Nonlinearly Perturbed Differential Systems with Multiple Delay. Journal of Applied Mathematics and Physics, 7, 30033011.

https://doi.org/10.4236/jamp.2019.712210

Received: November 6, 2019

Accepted: December 6, 2019

Published: December 9, 2019

Copyright () 2019 by author(s) and Scientific Research Publishing Inc. This work is licensed under the Creative Commons Attribution International License (CC BY 4.0).

http://creativecommons.org/licenses/by/4.0/

\begin{abstract}
In this paper, firstly a new class of time-delay differential inequality is proved. Then as an application, the nonlinearly perturbed differential systems with multiple delay are considered and it is obtained that the trivial solution of the nonlinear systems with multiple delay has uniform stability and uniform exponential Lipschitz asymptotic stability with respect to partial variables. It is obvious that the above system is a generalization of the traditional differential systems. The aim of this paper is to investigate the double stability of time-delay differential equations, including Uniform stability and Uniform Lipschitz stability. The author uses the method of differential inequalities with time-delay and integral inequalities to establish double stability criteria. As a result, the partial stability of differential equations is widely used both in theory and in practice such as dynamic systems and control systems.
\end{abstract}

\section{Keywords}

Time-Delay, Nonlinear Systems, Double Stability, Differential Inequality, Integral Inequality

\section{Introduction}

In 1892, Lyapunov, a Russian mathematician, mechanician and physicist, proposed the notion of the stability of motion. He gave the general research methods in his doctoral dissertation "The general problem of the stability of motion" [1], in which he established the foundation of the stability theory. When studying nonlinear systems, especially studying dynamic systems or control systems, we cannot study the stability of all variables because of the technology dif- 
ficulties, the limitation of practical conditions, or it is not necessary to study all variables considering the actual need. As a result, studying the partial stability of differential equations becomes more important. In addition, the partial stability is widely used in science and technology. For instance, the absolute stability of famous Lurie adjusting systems can be changed into a problem of partial stability. In a word, it is of practical significance to study the partial stability of differential equations.

In 1986, Dannan and Elaydi ([2]) introduced a new notion of stability, which is called uniform Lipschitz stability (ULS), for systems of differential equations

$$
\frac{\mathrm{d} x}{\mathrm{~d} t}=f(t, x),
$$

where $f \in C\left[J \times R^{n}, R^{n}\right], \quad J=[0, \infty), \quad f(t, 0)=0$, and $x\left(t, t_{0}, x_{0}\right) \equiv x(t)$ is the solution of (1) with $x\left(t_{0}, t_{0}, x_{0}\right)=x_{0}$, where $t_{0} \geq 0$.

This notion of ULS lies somewhere between uniform stability (US) on one side and the notions of asymptotic stability in variation (ASV) and uniform stability in variation (USV) on the other side. An important feature of ULS is that the linearized system inherits the property of ULS from the original nonlinear system.

YU-LI Fu ([3]) considers the system with time-delay

$$
\frac{\mathrm{d} x}{\mathrm{~d} t}=f\left(t, x_{t}\right)
$$

where $x \in R^{n}, f: R \times C\left([-r, 0], R^{n}\right) \mapsto R^{n}, f(t, 0)=0, f$ is continuous, $x_{t}=x(t+\theta), \theta \in[-r, 0], r>0$.

Sung Kyu Choi, Ki Shik Koo and Keonhee Lee ([4]) investigated the problems of ULS, EAS and GEASV for the following various perturbed differential systems of the nonlinear differential system (1) and

$$
\begin{gathered}
\frac{\mathrm{d} x}{\mathrm{~d} t}=A(t) x+g(t, x), \\
\frac{\mathrm{d} x}{\mathrm{~d} t}=f(t, x)+g(t, x),
\end{gathered}
$$

where $A(t)$ is a continuous $n \times n$ matrix defined on $R^{+}$, $g(t, x) \in C\left(R^{+} \times R^{n}, R^{n}\right)$ with $g(t, 0)=0$.

Vorotnikov, V. I. ([5] [6]) considered the following system

$$
\left\{\begin{array}{l}
\frac{\mathrm{d} y}{\mathrm{~d} t}=A(t) y+B(t) z+Y(t, y, z) \\
\frac{\mathrm{d} z}{\mathrm{~d} t}=C(t) y+D(t) z+Z(t, y, z)
\end{array},\right.
$$

and studied the double stability as $\|y\|+\|z\| \rightarrow 0$ and

$$
\frac{\|Y(t, y, z)\|+\|Z(t, y, z)\|}{\|y\|+\|z\|} \rightarrow 0 .
$$

In this paper, the author considers a new class of the nonlinearly perturbed 
differential systems with time-delay

$$
\frac{\mathrm{d} x}{\mathrm{~d} t}=A(t) x+f\left(t, x(t), x(t-\tau), \int_{0}^{t} h(s, x(s), x(s-\tau)) \mathrm{d} s\right),
$$

where $x \in R^{n}, y=\operatorname{col}\left(x_{1}, x_{2}, \cdots, x_{m}\right), z=\operatorname{col}\left(x_{m+1}, x_{m+2}, \cdots, x_{n}\right)$, $x=\operatorname{col}(y, z), \quad f: R \times R^{n} \times C\left([-r, 0], R^{n}\right) \times R^{n} \mapsto R^{n}, \quad f(t, 0,0,0) \equiv 0$, $h: R \times R^{n} \times C\left([-r, 0], R^{n}\right) \mapsto R^{n}, \tau$ is a non-negative constant.

It is obvious that the above system is a generalization of the systems in [2]-[6]. The aim of this paper is to investigate the double stability of time-delay differential equations, including Uniform stability and Uniform Lipschitz stability. The author uses the method of differential inequalities with time-delay and integral inequalities to establish double stability criteria.

\section{Preliminaries}

\section{1) Definitions and lemmas}

Consider the following system:

$$
\frac{\mathrm{d} x}{\mathrm{~d} t}=f(t, x, x(t-\tau)),
$$

where $x \in R^{n}, y=\operatorname{col}\left(x_{1}, x_{2}, \cdots, x_{m}\right), z=\operatorname{col}\left(x_{m+1}, x_{m+2}, \cdots, x_{n}\right)$, $x=\operatorname{col}(y, z), \quad f(t, 0,0) \equiv 0, \tau$ is a non-negative constant. Let $\phi(t)$ be a continuous function, for $\forall t \in E_{t_{0}}=\left[t_{0}-\tau, t_{0}\right]$.

Definition 1: The trivial solution of system (7) has uniform stability and exponential asymptotic stability with respect to $y$ if, for $\forall \varepsilon>0, \forall t_{0} \in I$, $\exists \delta(\varepsilon)>0$ and $\lambda>0$, when $\|\phi\|<\delta$ (for $\forall t \in E_{t_{0}}$ ), such that $\left\|x\left(t ; t_{0}, \phi\right)\right\|<\varepsilon,\left\|y\left(t ; t_{0}, \phi\right)\right\|<\varepsilon \exp \left(-\lambda\left(t-t_{0}\right)\right)$, for all $t \geq t_{0}$.

Definition 2: The trivial solution of system (7) has Lipschitz stability with respect to $y$ if, there exist constants $M\left(t_{0}\right)>0$ and $\delta\left(t_{0}\right)>0$, when $\|\phi\|<\delta$ (for $\forall t \in E_{t_{0}}$ ), such that $\left\|y\left(t ; t_{0}, \phi\right)\right\| \leq M\left(t_{0}\right)\|\phi\|$, for all $t \geq t_{0} \geq 0$.

Definition 3: The trivial solution of system (7) has equi-exponential Lipschitz asymptotic stability with respect to $y$ if, there exist constants $\lambda>0, K\left(t_{0}\right)>0$ and $\delta\left(t_{0}\right)>0$, when $\|\phi\|<\delta$ (for $\forall t \in E_{t_{0}}$ ), such that $\left\|y\left(t ; t_{0}, \phi\right)\right\| \leq K\left(t_{0}\right)\|\phi\| \exp \left(-\lambda\left(t-t_{0}\right)\right)$, for all $t \geq t_{0} \geq 0$.

Definition 4: The trivial solution of system (7) has uniform exponential Lipschitz asymptotic stability with respect to $y$ if, $K$ and $\delta$ in Definition3 are unrelated to $t_{0}$.

Lemma 1. [7] Consider the homogeneous system

$$
\left\{\begin{array}{l}
\frac{\mathrm{d} y}{\mathrm{~d} x}=B(t) y+C(t) z \\
\frac{\mathrm{d} z}{\mathrm{~d} x}=D(t) y+E(t) z
\end{array},\right.
$$

if the trivial solution of system (8) has uniform stability, and has exponential asymptotic stability with respect to $y$, then there exists a Lyapunov-function $V(t, x)$ satisfied the following conditions:

$$
\|y\| \leq V(t, x) \leq M\|x\|,\left.\dot{V}\right|_{(8)} \leq-\alpha V(t, x),
$$


where $M>0$.

Consider the following inequality:

$$
\dot{x}_{i}(t) \leq f_{i}(t)\left[-r_{i} x_{i}(t)+h_{i}^{(1)}\left(x_{t}\right) x_{t}^{\alpha_{i}}+\int_{-\infty}^{t} h_{i}^{(2)}(t-s, x(s)) x^{\beta_{i}}(t) \mathrm{e}^{-\varepsilon(t-s)} \mathrm{d} s\right],
$$

where $f_{i}(t) \in C\left[R, R_{+}\right]$and $f_{i}(t) \geq \beta=$ const $>0, \quad r_{i}=$ const $>0$, $(i=1,2, \cdots, m), h_{i}^{(1)}(\cdot), h_{i}^{(2)}(t-\theta, \cdot)$ are nonnegative and not monotone decreasing for “. ", $\alpha_{i}, \beta_{i} \geq 1, x(\theta) \triangleq \max _{1 \leq j \leq n}\left(x_{j}(\theta)\right), \quad x_{t} \triangleq \max _{1 \leq j \leq n}\left(\sup _{t-\tau \leq \theta \leq t} x_{j}(\theta)\right)$, $\tau=$ const $>0, \alpha \triangleq \max \left(\alpha_{i}, \beta_{i}\right)$.

Lemma 2. [8] Suppose $x_{i}(t)$ be nonnegative continuous on $R_{+}$, for all $t \geq t_{0}$ (3) is satisfied, if $\exists K=$ const the following inequality holds:

$$
h_{i}^{(1)}(K)+\int_{0}^{+\infty} h_{i}^{(2)}(s, K) \mathrm{d} s<r_{i}, \alpha K^{1-\frac{1}{\alpha}}<1,
$$

when $M^{\alpha} \triangleq \max _{1 \leq j \leq n}\left(\sup _{t_{0}-\tau \leq \theta \leq t_{0}} x_{j}(\theta)\right)<K$, we have following result:

$$
x_{i}(t) \leq M \exp \left(-\lambda\left(t-t_{0}\right)\right),
$$

holds true, where $t \geq t_{0}$, and $\lambda>0$.

2) Differential Inequalities with Time-Delay

Consider the following inequality

$$
\dot{x}_{i}(t) \leq f_{i}(t)\left[-r_{i} x_{i}(t)+h_{i}^{(1)}\left(x_{t}\right) x_{t}^{\alpha_{i}}+\int_{-\infty}^{t} h_{i}^{(2)}(t-s, x(s)) x^{\beta_{i}}(t) \mathrm{e}^{-\varepsilon(t-s)} \mathrm{d} s\right],
$$

where $f_{i}(t) \in C\left[R, R^{+}\right]$and $f_{i}(t) \geq \gamma=$ const $>0, r_{i}=$ const $>0, h_{i}^{(1)}(\cdot)$, $h_{i}^{(2)}(t-\theta, \cdot) \quad(i=1,2, \cdots, n)$ are nonnegative and not monotone ecreasing for “. ", $\quad \alpha_{i}, \beta_{i} \geq 1, x(\theta)=\max _{1 \leq i \leq n}\left(x_{i}(\theta)\right), \quad x_{t}=\max _{1 \leq i \leq n}\left(\sup _{t-\tau<\theta<t} x_{i}(\theta)\right), \quad \tau=$ const $>0$, $\alpha=\max \left(\alpha_{i}, \beta_{i}\right)$.

Lemma 3. Assume $x_{i}(t)$ be nonnegative continuous on $R_{+},(10)$ is satisfied for all $t \geq t_{0}$, there exists a constant $K$ satisfied the following inequality:

$$
h_{i}^{(1)}(K)+\int_{0}^{+\infty} h_{i}^{(2)}(s, K) \mathrm{d} s<r_{i},
$$

and

$$
\alpha K^{1-\frac{1}{\alpha}}<1
$$

then if $M^{\alpha} \triangleq \max _{1 \leq i \leq n}\left(\sup _{t_{0}-\tau \leq \theta \leq t_{0}} x_{i}(\theta)\right)<K$, the following inequality:

$$
x_{i}(t) \leq M \exp \left(-\lambda\left(t-t_{0}\right)\right) \quad(i=1,2, \cdots, n)
$$

holds true, where $t \geq t_{0}$ and $\lambda>0$.

\section{Proof}

According to (10), for $\forall \varepsilon>0, \exists \lambda$ (let $\lambda<\frac{\varepsilon}{\alpha}$ ) we can get 


$$
-r_{i}+\frac{\alpha \lambda}{\gamma}+\mathrm{e}^{\alpha \lambda \tau} h_{i}^{(1)}(K)+\int_{0}^{+\infty} h_{i}^{(2)}(s, K) \mathrm{d} s<0 .
$$

Now define

$$
P_{i}(t) \triangleq\left\{\begin{array}{l}
x_{i}^{\alpha}(t) \mathrm{e}^{\alpha \lambda\left(t-t_{0}\right)}, t \geq t_{0} ; \quad(12) \quad(i=1,2, \cdots, n) \\
x_{i}^{\alpha}(t), t \in\left[t_{0}-\tau, t_{0}\right],
\end{array}\right.
$$

thus, we can have

$$
x_{i}(t)=P_{i}^{\frac{1}{\alpha}}(t) \mathrm{e}^{-\lambda\left(t-t_{0}\right)}
$$

furthermore

$$
\dot{x}_{i}(t)=\left(\frac{1}{\alpha} P_{i}^{\frac{1}{\alpha}-1}(t) \dot{P}_{i}(t)-\lambda P_{i}^{\frac{1}{\alpha}}(t)\right) \mathrm{e}^{-\lambda\left(t-t_{0}\right)} .
$$

Let

$$
P_{t}=\max _{1 \leq i \leq n}\left(\sup _{t-\tau<\theta<t} P_{i}(\theta)\right), \quad P(\theta)=\max _{1 \leq i \leq n}\left(P_{i}(\theta)\right),
$$

obviously

$$
P_{t} \geq x_{t}^{\alpha}, \quad P(\theta) \geq x^{\alpha}(\theta)
$$

hence

$$
\begin{aligned}
\dot{x}_{i}(t) & =\left(\frac{1}{\alpha} P_{i}^{\frac{1}{\alpha}-1}(t) \dot{P}_{i}(t)-\lambda P_{i}^{\frac{1}{\alpha}}(t)\right) \mathrm{e}^{-\lambda\left(t-t_{0}\right)} \\
& \leq f_{i}(t)\left[-r_{i} x_{i}(t)+h_{i}^{(1)}\left(x_{t}\right) x_{t}^{\alpha_{i}}+\int_{-\infty}^{t} h_{i}^{(2)}(t-s, x(s)) x^{\beta_{i}}(t) \mathrm{e}^{-\varepsilon(t-s)} \mathrm{d} s\right] .
\end{aligned}
$$

Notice that

$$
\begin{aligned}
x_{t}^{\alpha} & =\max _{1 \leq i \leq n}\left(\sup _{t-\tau<\theta<t} x_{i}^{\alpha}(\theta)\right) \\
& =\max _{1 \leq i \leq n}\left(\sup _{t-\tau<\theta<t} x_{i}^{\alpha}(\theta)\right) \\
& =\max _{1 \leq i \leq n}\left(\sup _{t-\tau<\theta<t} P_{i}(\theta) \mathrm{e}^{-\alpha \lambda\left(\theta-t_{0}\right)}\right) \\
& =\max _{1 \leq i \leq n}\left(\sup _{t-\tau<\theta<t} P_{i}(\theta)\right) \mathrm{e}^{-\alpha \lambda\left(t-\tau-t_{0}\right)} \\
& =P_{t} \mathrm{e}^{-\alpha \lambda\left(t-t_{0}\right)} \mathrm{e}^{\alpha \lambda \tau},
\end{aligned}
$$

and

$$
\begin{aligned}
x^{\alpha}(\theta) & =\max _{1 \leq i \leq n}\left(x_{i}^{\alpha}(\theta)\right) \\
& =\max _{1 \leq i \leq n}\left(P_{i}(\theta) \mathrm{e}^{-\alpha \lambda\left(\theta-t_{0}\right)}\right) \\
& =\max _{1 \leq i \leq n}\left(P_{i}(\theta) \mathrm{e}^{-\alpha \lambda\left(\theta-t_{0}\right)}\right) \\
& =\max _{1 \leq i \leq n}\left(P_{i}(\theta)\right) \mathrm{e}^{-\alpha \lambda\left(\theta-t_{0}\right)} \\
& =P(\theta) \mathrm{e}^{-\alpha \lambda\left(\theta-t_{0}\right)} .
\end{aligned}
$$

Applying (12) into (10), we have 


$$
\begin{aligned}
\dot{P}_{i}(t) \leq & f_{i}(t)\left[-\left(r_{i}-\frac{\alpha \lambda}{\gamma}\right) P_{i}(t)+\left(h_{i}^{(1)}\left(x_{t}\right) P_{t} \mathrm{e}^{\alpha \lambda \tau}\right.\right. \\
& \left.\left.+\int_{0}^{+\infty} h_{i}^{(2)}(t-s, x(s)) P(s) \mathrm{d} s\right) \alpha P_{i}^{1-\frac{1}{\alpha}}(t)\right] .
\end{aligned}
$$

For any scaler $l \in\left(1, \frac{K}{M^{\alpha}}\right)$, we can get

$$
P_{i}(t) \leq l M^{\alpha} \triangleq N
$$

If not, then $P_{i}(t)<N$, thus there exists a certain $i$ in $\left(-\infty, t_{0}\right]$ and $t_{1}>t_{0}$, we have

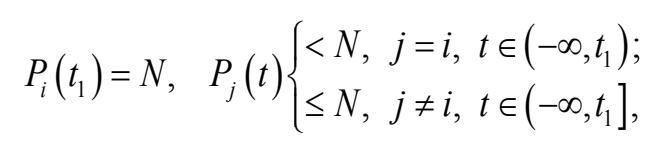

thus we can get $\dot{P}_{i}\left(t_{1}\right) \geq 0$. Using it in (14), we get

$$
\begin{aligned}
\dot{P}_{i}\left(t_{1}\right) \leq & f_{i}\left(t_{1}\right)\left[-\left(r_{i}-\frac{\alpha \lambda}{\beta}\right) P_{i}\left(t_{1}\right)+\left(h_{i}^{(1)}\left(x_{t_{1}}\right) P_{t_{1}} \mathrm{e}^{\alpha \lambda \tau}\right.\right. \\
& \left.\left.+\int_{-\infty}^{t_{1}} h_{i}^{(2)}\left(t_{1}-s, x(s)\right) P(s) \mathrm{d} s\right) \alpha P_{i}^{1-\frac{1}{\alpha}}\left(t_{1}\right)\right] \\
\leq & f_{i}\left(t_{1}\right)\left[-\left(r_{i}-\frac{\alpha \lambda}{\beta}\right) K+\left(h_{i}^{(1)}(K) K \mathrm{e}^{\alpha \lambda \tau}+\int_{0}^{+\infty} h_{i}^{(2)}(s, K) K \mathrm{~d} s\right) \alpha K^{1-\frac{1}{\alpha}}\right] \\
\leq & f_{i}\left(t_{1}\right)\left[-\left(r_{i}-\frac{\alpha \lambda}{\beta}\right)+\left(h_{i}^{(1)}(K) \mathrm{e}^{\alpha \lambda \tau}+\int_{0}^{+\infty} h_{i}^{(2)}(s, K) \mathrm{d} s\right)\right] K \\
< & 0 .
\end{aligned}
$$

It is a contradictory, thus $P_{i}(t) \leq l M^{\alpha}$, let $l \rightarrow 1$, we can get

$$
P_{i}(t) \leq M^{\alpha} .
$$

Notice (12), the following is obtained

$$
x_{i}(t) \leq M \exp \left(-\lambda\left(t-t_{0}\right)\right) \text {, for all } t \geq t_{0} .
$$

Remark It is obvious that when $\alpha_{i}=1, \beta_{i}=1$ lemma 2 can be deduced by lemma 3.

\section{Main Results}

Consider the following system which is equivalent with system (1)

$$
\begin{gathered}
\left\{\begin{array}{l}
\frac{\mathrm{d} y}{\mathrm{~d} t}=B(t) y+C(t) z+Y\left(t, y(t), z(t), \int_{0}^{t} h_{1}(s, y(s), z(s), y(s-\tau), z(s-\tau)) \mathrm{d} s\right) \\
\frac{\mathrm{d} x}{\mathrm{~d} t}=D(t) y+E(t) z+Z\left(t, y(t), z(t), \int_{0}^{t} h_{2}(s, y(s), z(s), y(s-\tau), z(s-\tau)) \mathrm{d} s\right)
\end{array}\right. \\
\text { where } x=(y, z)^{\mathrm{T}}, \tau \geq 0 \text { is a constant, initial condition is: }
\end{gathered}
$$

$$
x(t)=\varphi(t), t_{0}-\tau \leq t \leq t_{0},
$$

$$
B(t) \text { is an } m \times m \text { matrix, }
$$


$Y\left(t, y(t), z(t), \int_{0}^{t} h_{1}(s, y(s), z(s), y(s-\tau), z(s-\tau)) \mathrm{d} s\right) \quad$ is an $m \times 1$ matrix,
$Z\left(t, y(t), z(t), \int_{0}^{t} h_{2}(s, y(s), z(s), y(s-\tau), z(s-\tau)) \mathrm{d} s\right)$ is an $(n-m) \times 1$ matrix, they are all continuous for $t \in I$ and satisfy the condition of existence and uniqueness theorem.

The homogeneous system of (15) is

$$
\left\{\begin{array}{l}
\frac{\mathrm{d} y}{\mathrm{~d} t}=B(t) y+C(t) z \\
\frac{\mathrm{d} x}{\mathrm{~d} t}=D(t) y+E(t) z
\end{array} .\right.
$$

Theorem: If (15) satisfies the following conditions:

1) $Y(t, 0,0,0,0,0) \equiv Y(t, 0, z, 0, z(t-\tau), 0) \equiv 0$.

2) $Z(t, 0,0,0,0,0) \equiv Z(t, 0, z, 0, z(t-\tau), 0) \equiv 0$. $\left\|Y\left(t, y, z, y(t-\tau), z(t-\tau), \int_{0}^{t} h_{1}(s, y, z, y(s-\tau), z(t-\tau)) \mathrm{d} s\right)\right\|$

3)

$$
\begin{aligned}
& +\left\|Z\left(t, y, z, y(t-\tau), z(t-\tau), \int_{0}^{t} h_{1}(s, y, z, y(s-\tau), z(t-\tau)) \mathrm{d} s\right)\right\| \\
& \leq r\|y\|+h^{(1)}\left(\|y(t-\tau)\|^{\alpha}\right)\|y(t-\tau)\|^{\alpha} \\
& +\int_{-\infty}^{t} h^{(2)}\left(t-s,\|y(s)\|^{\alpha}\right)\|y(s)\|^{\alpha} \mathrm{e}^{-\varepsilon(t-s)} \mathrm{d} s
\end{aligned}
$$

where $h^{(1)}(\cdot), h^{(2)}(t-\theta, \cdot)$ are nonnegative and not monotone decreasing for “.", $\alpha \geq 1$, and

$$
h^{(1)}(K)+\int_{0}^{+\infty} h^{(2)}(s, K) \mathrm{d} s<r, \alpha K^{1-\frac{1}{\alpha}} \leq 1,
$$

then the trivial solution of system (15) has uniform exponential Lipschitz asymptotic stability with respect to $y$, when the trivial solution of system (15)* has uniform stability and exponential asymptotic stability with respect to $y$.

Proof The V-Ляпунов function of $(15)^{*}$, which is obtained under the condition of theorem, satisfies following conditions:

$$
\begin{gathered}
\|y\| \leq V(t, x) \leq M\|x\|, \quad \dot{V}_{(15)^{*}}(t, x) \leq-a V(t, x), \\
\left|V\left(t, x^{\prime \prime}\right)-V\left(t, x^{\prime}\right)\right| \leq M\left\|x^{\prime \prime}-x^{\prime}\right\|, \quad(a, M=\text { const }>0),
\end{gathered}
$$

for $t \geq 0,\|x\|<\infty$.

Derivative the V-Ляпунов function $V(t, x)$ along (15), we get

$$
\dot{V}_{(15)}(t, x) \leq-a V(t, x)+R\left(t, x(t), x(t-\tau), \int_{0}^{t} h(s, x(s), x(s-\tau)) \mathrm{d} s\right),
$$

where

$$
\begin{aligned}
& R\left(t, x(t), x(t-\tau), \int_{0}^{t} h(s, x(s), x(s-\tau)) \mathrm{d} s\right) \\
& =\left\langle\frac{\partial V}{\partial x}, X^{*}\left(t, x(t), x(t-\tau), \int_{0}^{t} h(s, x(s), x(s-\tau)) \mathrm{d} s\right)\right\rangle,
\end{aligned}
$$




$$
\begin{aligned}
X^{*}\left(t, x(t), x(t-\tau), \int_{0}^{t} h(s, x(s), x(s-\tau)) \mathrm{d} s\right) \\
=\left\{Y\left(t, x(t), x(t-\tau), \int_{0}^{t} h(s, x(s), x(s-\tau)) \mathrm{d} s\right),\right. \\
\left.Z\left(t, x(t), x(t-\tau), \int_{0}^{t} h(s, x(s), x(s-\tau)) \mathrm{d} s\right)\right\}^{\mathrm{T}}
\end{aligned}
$$

here $\langle$,$\rangle the notation of inner product.$

From condition of theorem and (17), when $t \geq t_{0}$ we have

$$
\begin{aligned}
& R\left(t, x(t), x(t-\tau), \int_{0}^{t} h(s, x(s), x(s-\tau)) \mathrm{d} s\right) \\
& \leq M\left[r\|y(t)\|+h^{(1)}\left(\|y(t-\tau)\|^{\alpha}\right)\|y(t-\tau)\|^{\alpha}\right. \\
& \left.\quad+\int_{-\infty}^{t} h^{(2)}\left(t-s,\|y(s)\|^{\alpha}\right)\|y(s)\|^{\alpha} \mathrm{e}^{-\varepsilon(t-s)} \mathrm{d} s\right] .
\end{aligned}
$$

By the first inequality of (16), the above can be expressed as follow:

$$
\begin{aligned}
& R\left(t, x(t), x(t-\tau), \int_{0}^{t} h(s, x(s), x(s-\tau)) \mathrm{d} s\right) \\
& \leq M\left[r V(t, x)+h^{(1)}\left(V^{\alpha}(t, x)\right) V^{\alpha}(t, x)\right. \\
& \left.\quad+\int_{-\infty}^{t} h^{(2)}\left(t-s, V^{\alpha}(t, x)\right) V^{\alpha}(t, x) \mathrm{e}^{-\varepsilon(t-s)} \mathrm{d} s\right],
\end{aligned}
$$

then there exists $K>0$ such that when $t \geq t_{0}$ and

$$
\begin{aligned}
& \sup _{t_{0}-\tau \leq \sigma \leq t_{0}}\left\{V\left(\sigma, x\left(\sigma, t_{0}, \phi\right)\right)\right\}<K \text {, we get } \\
& \begin{aligned}
\dot{V}_{(15)}(t, x) \leq & -a V(t, x)+M\left[r V(t, x)+h^{(1)}\left(V^{\alpha}(t, x)\right) V^{\alpha}(t, x)\right. \\
& \left.+\int_{-\infty}^{t} h^{(2)}\left(t-s, V^{\alpha}(t, x)\right) V^{\alpha}(t, x) \mathrm{e}^{-\varepsilon(t-s)} \mathrm{d} s\right], \\
= & M\left[-\left(\frac{a}{M}-r\right) V(t, x)+h^{(1)}\left(V^{\alpha}(t, x)\right) V^{\alpha}(t, x)\right]
\end{aligned},
\end{aligned}
$$

here select the appropriate small constant $r$ such that

$$
r^{\prime}=\frac{a}{M}-r>0 \text { and } h^{(1)}(K)+\int_{0}^{+\infty} h^{(2)}(s, K) \mathrm{d} s<r^{\prime},
$$

hence by the lemma [9] [10], there exists $\lambda>0$ such that for all $t \geq t_{0}$ we have

$$
V(t, x)<\sup _{t_{0}-\tau \leq \sigma \leq t_{0}}\left\{V\left(\sigma, x\left(\sigma, t_{0}, \phi\right)\right)\right\} \exp \left(-\lambda\left(t-t_{0}\right)\right) .
$$

For any solution of (15), from the inequality (18) and the first inequality of (16) we obtain

$$
\|y(t)\| \leq V(t, x) \leq M\|\phi\| \exp \left(-\lambda\left(t-t_{0}\right)\right) .
$$

According to the proof of the theorem in [11], we get $\|x\|<\varepsilon$, hence we obtain that the trivial solution of system (15) has uniform stability and uniform exponential Lipschitz asymptotic stability with respect to $y$.

\section{Conclusion}

In this paper, we use the method of differential inequalities with time-delay and 
integral inequalities to establish double stability criteria. As a result, studying the partial stability of differential equations becomes more important. In addition, the partial stability of differential equations is widely used in science and technology.

\section{Acknowledgements}

The authors are grateful to Professor Si Ligeng and the referee for several helpful comments.

\section{Supported}

Supported by Inner Mongolia Autonomous Region Higher Education Research Project (No.NJZY17064, NJZY16141).

\section{Conflicts of Interest}

The authors declare no conflicts of interest regarding the publication of this paper.

\section{References}

[1] Lyapunov, A.M. (1892) The General Problem of the Stability of Motion. Doctor Dissertation, University of Moscow, Moscow.

[2] Dannan, F.M. and Elaydi, S. (1986) Lipschitz Stability of Nonlinear Differential Equations. Journal of Mathematical Analysis and Applications, 113, 562-577. https://doi.org/10.1016/0022-247X(86)90325-2

[3] Fu, Y.L. (1991) On Lipschitz Stablity for F.D.E. Pacific Journal of Mathematics, 151, 229-235. https://doi.org/10.2140/pjm.1991.151.229

[4] Choi, S.K., Koo, K.S. and Lee, K. (1992) Lipschitz Stability and Exponential Asymptotic Stability in Perturbed Systems. Journal of the Korean Mathematical Society, 29, 175-190.

[5] Vorotnikov, V.I. (1999) On the Theory of Lyapunov Stability in Critical Cases. Doklady Akademii Nauk SSSR, 367, 481-484.

[6] Vorotnikov, V.I. (1999) Problems of Stability with Respect to Part of the Variables. Journal of Applied Mathematics and Mechanics, 63, 695-703. https://doi.org/10.1016/S0021-8928(99)00088-X

[7] Cordunmeanu, C. (1971) Some Problems Concerning Partial Stability. Sympos Math, 6, 141-154.

[8] Zhang, Y. (1994) K-Stablity of Nonlinear Systerm with Time-Delay. Scientia Sinica Mathematica, 37, 247-255.

[9] Si, L.G. (1974) The Boundness and Stability of Solutions for Nonlinear Neutral Differential Systems with Variable Delay. Acta Mathematica Sinica, 197-204.

[10] Pachpatte, B.G. (1978) On Some Generalizations of Bellman's Lemma. Journal of Mathematical Analysis and Applications, 51, 141-150. https://doi.org/10.1016/0022-247X(75)90146-8

[11] Huo, R., Zhang, C.Q., Wang, X.L. and Si, L.G. (2009) The Lipschitz Stability of a Kind of Nonlinear Delay Neutral Differential Systems Based on a New Integral Inequality. Mathematics in Practice and Theory, 39, 231-234. 